\title{
Hyper X-ray Flares on Active Stars Detected with MAXI
}

\author{
Masaya Higa ${ }^{1}$, Yohko Tsuboi ${ }^{1}$, Hitoshi Negoro ${ }^{2}$, Satoshi Nakahira ${ }^{3}$, \\ Hiroshi Tomida ${ }^{3}$, Masaru Matsuoka ${ }^{4}$ and The MAXI team ${ }^{2}$ \\ ${ }^{1}$ Department of Physics, Faculty of Science and Engineering, Chuo University \\ email: higa@phys.chuo-u.ac.jp \\ ${ }^{2}$ Department of Physics, Nihon University \\ ${ }^{3}$ Japan Aerospace Exploration Agency (JAXA) \\ ${ }^{4}$ MAXI team, RIKEN
}

\begin{abstract}
MAXI started its operation in 2009 August. Owing to its unprecedentedly high sensitivity as an all-sky X-ray monitor and to its capability of real-time data transfer, we have detected 56 strong flares from twenty-one active stars (eleven RS CVn systems, one Algol system, seven dMe stars, one dKe star and one Young Stellar Object). These flares have large X-ray luminosity of $6 \times 10^{30}-5 \times 10^{33} \mathrm{ergs} \mathrm{s}^{-1}$ in the $2-20 \mathrm{keV}$ band. The flares can be thought to be high ends among their own categories. During the flare from AT Mic on 2012 April 18th, one of the largest X-ray luminosities was recorded as a dMe star, $6 \times 10^{32} \mathrm{ergs} \mathrm{s}^{-1}$ in the $2-20 \mathrm{keV}$ band. It is larger than its bolometric luminosity by 4 times. The total energy emitted during the flare is $10^{36}$ ergs in the same band. Such total energy can be obtained on large flares from RS CVn system, but not on any other flares from dMe stars. In this proceeding, we report on the present situation in characteristics of hyper X-ray flares on each stellar categories.
\end{abstract}

Keywords. stars: flare - stars: RS-CVn type - stars: Algol - stars: dMe stars - stars: YSO

\section{Introduction}

Cool stars, which have spectral types of F, G, K, and M, are known to show X-ray flares (e.g., Favata \& Micela 2003; Güdel 2004). Generally, the larger flare occurs less frequently. Therefore, the observed sample (or number) of large flares is fairly limited, and the physical parameters such as the peak temperature, the emission measure $(E M)$, the $e$-folding time in the large flares have been poorly understood. This situation will be much improved if we can do un-biased all-sky survey with a large field of view and high sensitivity, which increases the chance to catch large flares.

The Monitor of All-Sky X-ray Image (MAXI; Matsuoka et al. 2009) is a mission of an all-sky X-ray monitor operated in the Japanese Experiment Module (JEM; Kibo) on the International Space Station (ISS) since 2009 August. It enables us to search for stellar flares effectively. In this proceeding, we report the results of survey for X-ray flares from stellar sources using the MAXI data taken by the first four-years operation from 2009 August to 2013 August.

\section{Observation}

The MAXI carries two scientific instruments: the Gas Slit Camera (GSC; Mihara et al. 2011; Sugizaki et al. 2011) covering the energy range of 2-30 keV and the Solid-state Slit Camera (SSC; Tsunemi et al. 2010; Tomida et al. 2011) covering that of $0.5-7 \mathrm{keV}$. This report is mainly based on the results obtained with the GSC, which has larger effective 

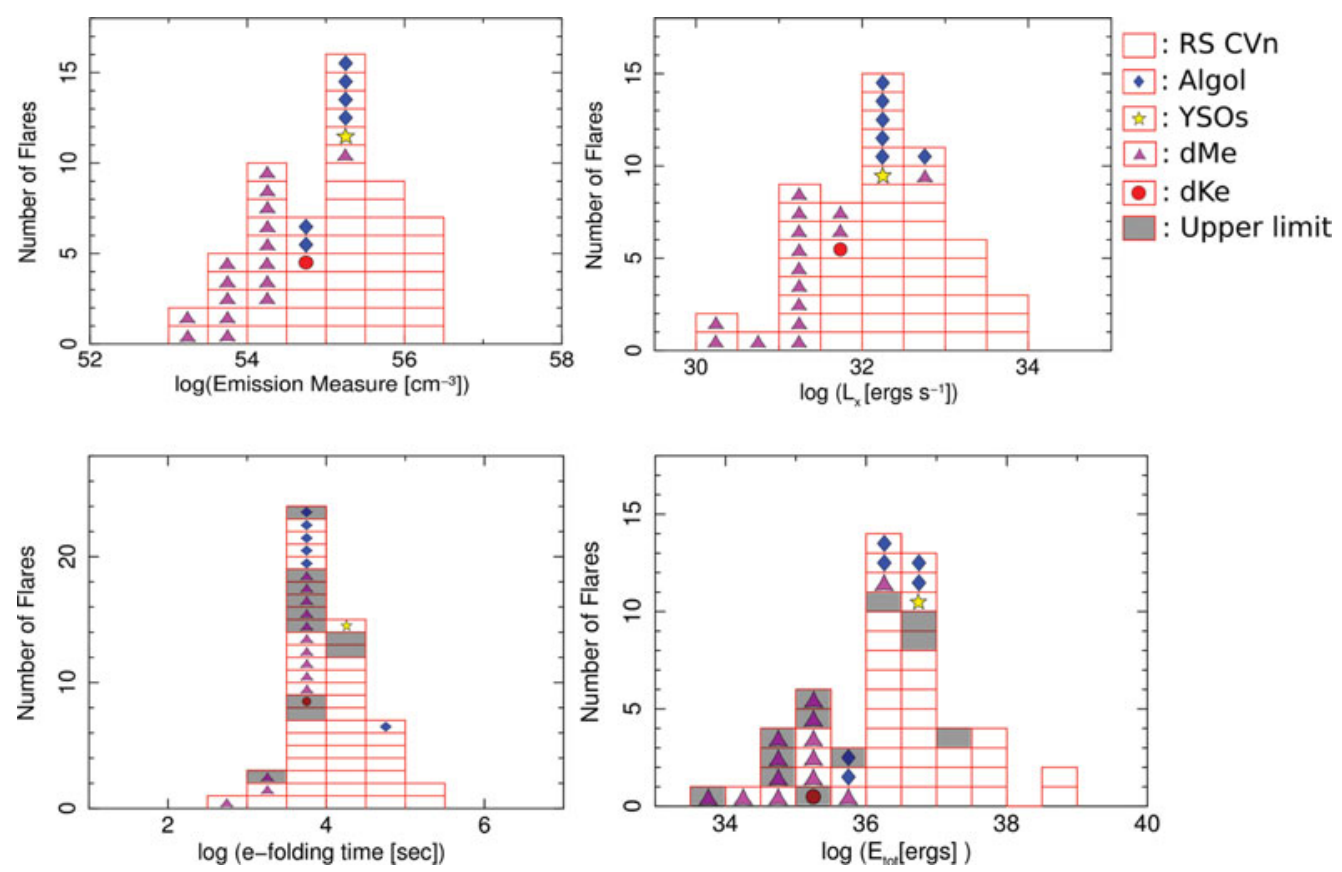

Figure 1. Distribution of the emission measure (top left), X-ray luminosity in the $2-20 \mathrm{keV}$ band (top right), $e$-folding time at the flare decaying phase (bottom left), and total energy released during the flare (bottom right). The total energy is derived by multiplying the $L_{\mathrm{X}}$ by the $e$-folding time. The open squares, filled diamonds, filled star, filled triangles, and filled circle show RS-CVn type stars, Algol, YSO, dMe stars and a dKe star, respectively. The shaded data mean upper limit.

area than the SSC, although the results of the dMe star AT Mic introduced here was mainly obtained with the SSC.

\section{Results}

We detected 56 large flares from twenty-one active stars (eleven RS CVn systems, one Algol system, seven dMe stars, one dKe star and one Young Stellar Object). The X-ray luminosities are higher than $10^{30} \mathrm{ergs} \mathrm{s}^{-1}$ in the $2-20 \mathrm{keV}$ band, and $E M$ s are higher than $10^{53} \mathrm{~cm}^{-3}$. These MAXI flares can be thought to be high ends among their own categories.

In figure 1, we show the distribution of the derived properties, $E M, L_{\mathrm{X}}, e$-folding time and $E_{\text {tot }}$. In the all the parameters, dMe flares are localized at smaller values than the RS CVn flares. As for the $E M, L_{\mathrm{X}}$, and $E_{\text {tot }}$, the high-ends in the flares from RS CVn stars are roughly two orders of magnitudes higher than those in the flares from dMe stars. The difference might originate from the area of starspots; RS CVn systems and dMe stars have similar coverage ratio of the starspots to the stellar surface, but the stellar radius of RS CVn stars is about one order of magnitude larger than that of dMe stars.

Among the flares of dMe stars, an outstanding flare was detected from AT Mic on 2012 April 18th. The $E M, L_{\mathrm{X}}$, and $E_{\text {tot }}$ are $3 \times 10^{55} \mathrm{~cm}^{-3}, 6 \times 10^{32} \mathrm{ergs} \mathrm{s}^{-1}$ in the 2-20 $\mathrm{keV}$ band, and $10^{36} \mathrm{ergs}$ in the same band, respectively, although the $e$-folding time is typical value for dMe stars, $2.6 \mathrm{ksec}$. The $L_{\mathrm{X}}$ is four times larger than $L_{\mathrm{bol}}$. 